\title{
Chemoenzymatic ortho-quinone methide formation
}

\author{
Tyler J. Doyon,,$^{1,2^{+}}$Jonathan C. Perkins,,$^{1,3^{+}}$Summer A. Baker Dockrey, ${ }^{1,3}$ Evan O. Romero, ${ }^{1,3}$ Kevin C. \\ Skinner,1,3 Paul M. Zimmerman, ${ }^{3}$ Alison R. H. Narayan ${ }^{1,2,3^{*}}$ \\ ${ }^{1}$ Life Sciences Institute, University of Michigan, Ann Arbor, Michigan 48109, USA. ${ }^{2}$ Program in Chemical Biology, Uni- \\ versity of Michigan, Ann Arbor, Michigan 48109, USA. ${ }^{3}$ Department of Chemistry, University of Michigan, Ann Arbor, \\ Michigan 48109, USA.
}

$\left.{ }^{+}\right]$These authors contributed equally to this work.

*Corresponding author, email: arhardin@umich.edu

KEYWORDS: biocatalysis, C-H functionalization, ortho-quinone methide

\begin{abstract}
Generation of reactive intermediates and interception of these fleeting species under physiological conditions is a common strategy employed by Nature to build molecular complexity. However, formation of these species under mild conditions using traditional synthetic techniques can present a challenge. Here, we demonstrate the utility of biocatalysis in generating ortho-quinone methide intermediates under aqueous conditions and at reduced temperatures. Specifically, we applied $\alpha$-ketoglutarate-dependent non-heme iron enzymes, CitB and ClaD, in the selective modification of benzylic C- $\mathrm{H}$ bonds of ortho-cresol substrates. In this transformation, these biocatalysts directly hydroxylate a benzylic $\mathrm{C}-\mathrm{H}$ bond to afford a benzylic alcohol product which, under the reaction conditions, is in equilibrium with the corresponding ortho-quinone methide. Interception of the ortho-quinone methide by a nucleophile or a dienophile allows for one-pot conversion of benzylic $\mathrm{C}-\mathrm{H}$ bonds into $\mathrm{C}-\mathrm{C}, \mathrm{C}-\mathrm{N}, \mathrm{C}-\mathrm{O}$, and $\mathrm{C}-\mathrm{S}$ bonds in a chemoenzymatic cascade. The versatility of this method is demonstrated through preparative-scale reactions, selective modification of peptides, and chemoenzymatic synthesis of the chroman natural product (-)-xyloketal D.
\end{abstract}

\section{INTRODUCTION}

Nature has developed strategies for the generation of highly reactive intermediates for the control of reactions in the synthesis of complex secondary metabolites.1-2 Synthetic approaches that mimic biosynthetic pathways often encounter challenges in reproducing the elegance of these evolved methods, at times requiring blocking groups to achieve site-selectivity, protection of sensitive functional groups, and harsh or forcing conditions to access reactive intermediates. ${ }^{3-6}$ Ortho-quinone methide species $(o-\mathrm{QM}, 4)$ have been implicated in the biosynthesis of multiple families of natural products, as these versatile intermediates can readily participate in inverse electron-demand Diels-Alder (IEDDA) or 1,4-addition reactions. ${ }^{3-5}$ These valuable and reactive intermediates have been used in the synthesis of a diverse array of natural products and bioactive compounds. ${ }^{3-}$ ${ }^{6}$ Several synthetic strategies have been developed to access $o$-QMs through the oxidative functionalization of phenolic substrates to generate $o-\mathrm{QM}$ precursors (Fig. 1A, 2-3). . $^{3-6}$ These methods typically involve the installation of a benzylic leaving group, followed by prolonged heating of the precursor to generate the corresponding $o-Q M .^{7-9}$ This route can also require super-stoichiometric reagents to activate the benzylic leaving-group, leading to reduced atom economy. ${ }^{7}$ Efforts to access $o$-QMs more efficiently have led to direct oxidation methods using transition metals or organic oxidants, to generate $o-\mathrm{QMs}$ in a one-pot system without the need for pre-functionalization of the substrate (Fig. 1A, 5-6)..$^{3-6,10-16}$ However, this approach is generally restricted to electron-rich compounds and does not provide control over the site-selectivity of the benzylic oxidation. ${ }^{3-6 \text {, }}$ 10-16 Furthermore, one-pot generation and functionalization of $o$-QMs requires a delicate balance between reactivity of the oxidant and compatibility with reagents for $o$-QM diversification. 3, 15 Often, the harsh oxidizing reagents which are required for $o-\mathrm{QM}$ generation can interfere with downstream functionalization of $o-\mathrm{QM}$ s or prove incompatible with sensitive moieties on the substrate. ${ }^{3}$ As a result, significant reagent screening campaigns are often necessary to optimize direct oxidation reactions, creating additional constraints on the application of this approach.3, 10-11, 13-15 Therefore, a one-pot method for the chemo- and site-selective generation and diversification of $o-\mathrm{QMs}$ would address key challenges in exploiting these useful synthetic intermediates in synthesis.

Inspired by the efficiency of Nature's approach for the generation of $o-\mathrm{QMs}$, we sought to exploit enzymatic mechanisms for $o-\mathrm{QM}$ creation. ${ }^{17}$ In these systems, the $o-\mathrm{QM}$ is proposed to arise from ortho-cresol precursors (see 7, 9, Fig. 1B) through hydroxylation of the benzylic position, followed by loss of water to directly form an $o$-QM under mild 
A. Small-molecule methods for oxidative generation of ortho-quinone methides

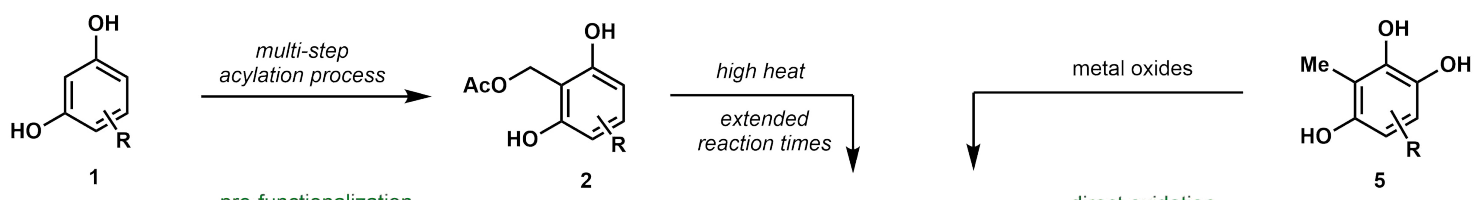

- multi-step installation of benzylic leaving group - high heat required for elimination to form O-QM - reduced selectivity for $\mathrm{O}-\mathrm{QM}$ diversification<smiles>Oc1cccc(O)c1</smiles>
process process
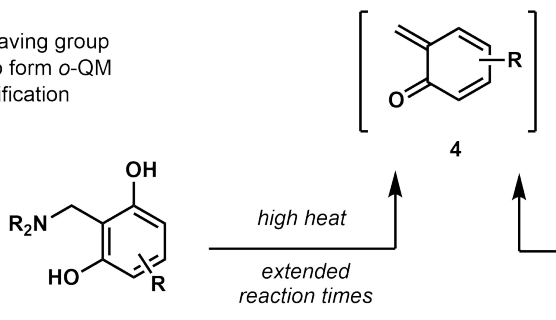

direct oxidation

- stoichiometric oxidants

- electron-rich substrates only

- some conditions incompatible with o-QM diversification

of oxidative benzylic functionalization in natural product biosynthesis

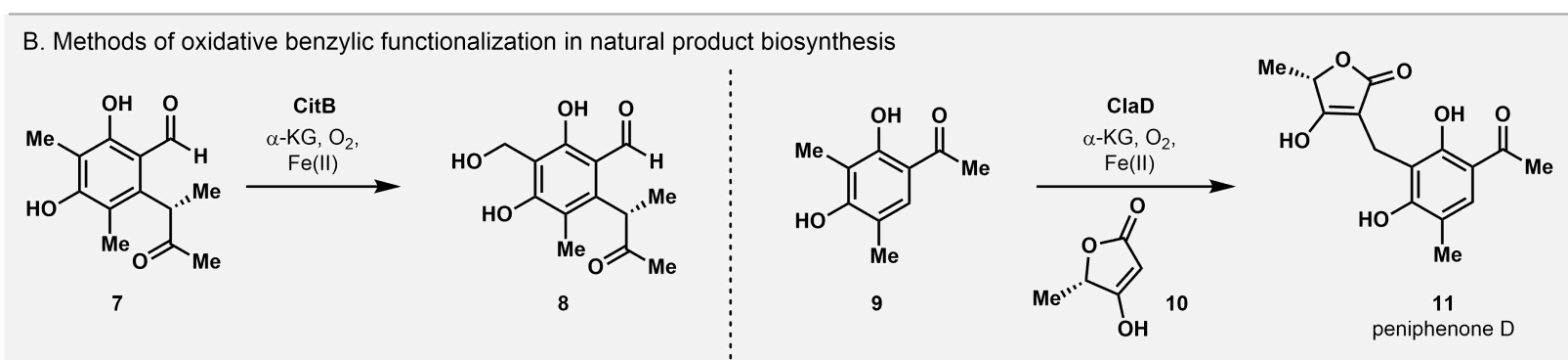

C. This work: One-pot biocatalyst-initiated ortho-quinone methide generation and diversification

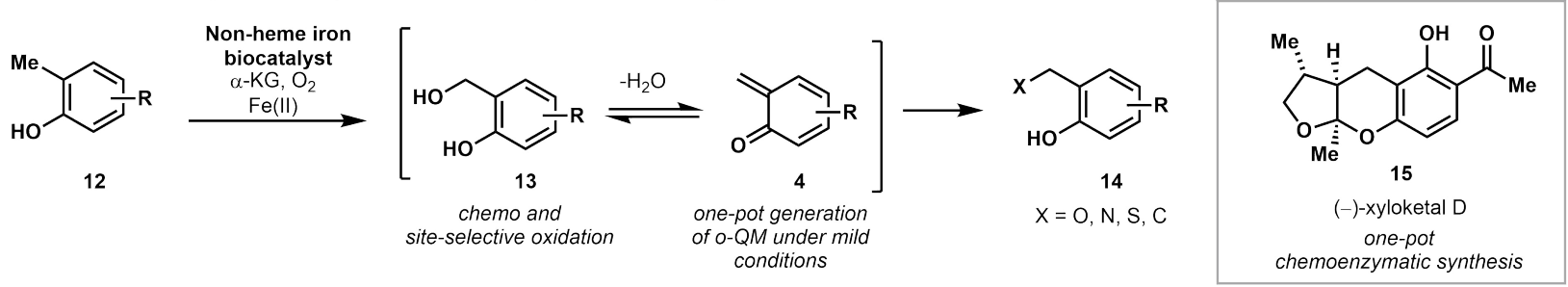

Figure 1. A. Oxidative methods for generation of ortho-quinone methides (o-QMs). B. Biocatalytic oxidative benzylic functionalization. C. This work: One-pot biocatalyst-initiated $o$-QM generation and diversification.

intracellular conditions. ${ }^{17}$ These intermediates are subsequently functionalized with bioavailable dienophiles or nucleophiles, generating the associated natural products, such as peniphenone $\mathrm{D}(\mathbf{1 1}) .{ }^{17}$ We anticipated that a biocatalytic approach to oxidative $o$-QM generation could afford numerous advantages in the development of a selective and sustainable method (Fig. 1C). This approach would combine the selectivity advantages of biocatalytic pre-functionalization with the ability to generate and diversify the $o$-QM in the same vessel under mild conditions. We also anticipated that the reactive nature of $o$-hydroxybenzylic alcohols would reduce required reaction times and temperatures, leading to efficient functionalization of the resulting $o-\mathrm{QM}$.

Nature has evolved numerous tools for the oxidation of benzylic $\mathrm{C}-\mathrm{H}$ bonds. Several reports have highlighted the utility of whole microorganism biotransformations in accomplishing this challenging transformation. ${ }^{18-23}$ These methods rely on native monooxygenases present in microbes to accomplish benzylic oxidation on a range of substrates. ${ }^{18-23}$ Several cytochromes $\mathrm{P} 450$ have also been identified as promising in vitro catalysts for benzylic $\mathrm{C}-\mathrm{H}$ oxygenation. ${ }^{24-32}$ Whereas these biocatalytic methods do not offer complete control over the final oxidation state of the product, they successfully reduce the overall environmental impact of the transformation by using earth-abundant iron as the catalytic metal, molecular oxygen as the stoichiometric oxidant, and water as the bulk solvent. ${ }^{24-32}$

Motivated by the advantages of an enzymatic approach to benzylic hydroxylation, we sought to demonstrate the siteand chemoselectivity of biocatalytic $\mathrm{C}-\mathrm{H}$ functionalization and opportunities for cascade reactions under biocatalytic reaction conditions. We anticipated that an $\alpha$-ketoglutaratedependent $(\alpha-\mathrm{KG})$ non-heme iron oxygenase could be employed for the selective oxidation of primary benzylic $\mathrm{C}-\mathrm{H}$ bonds. This well-studied family of enzymes has precedent for both scalability ${ }^{33}$ and substrate scope tunability through protein engineering. ${ }^{34}$

By coupling the activation of molecular oxygen to the decarboxylation of inexpensive $\alpha$-oxoacid co-substrates, these enzymes generate an iron(IV)-oxo species capable of $\mathrm{H}$ atom abstraction (Fig. 1C). ${ }^{35-36} \alpha$-KG-dependent non- heme iron oxygenases catalyze a wide variety of site-selective transformations following this conserved mechanism of oxygen activation, including hydroxylation, halogenation, epoxidation, desaturation, epimerization, endo-peroxidation, ring-contraction, and ring-expansion. ${ }^{35-37}$ Unlike many P450s, $\alpha$-KG-dependent non-heme iron oxygenases do not 
require an exogenous reductase to complete their catalytic cycle. ${ }^{35-36}$ We anticipated that the relatively simple reaction requirements of $\alpha$-KG-dependent non-heme iron oxygenases would provide distinct advantages in developing a widely applicable and practical approach to biocatalytic benzylic $\mathrm{C}-\mathrm{H}$ hydroxylation and subsequent $o-\mathrm{QM}$ generation.

Our search for biocatalysts for selective benzylic C-H hydroxylation led us to two homologous fungal NHI oxygenases (sharing 54\% sequence identity): (1) CitB, from Monascus ruber and (2) ClaD, native to Penicillium crustosum. ${ }^{38}$ Cox and coworkers demonstrated through in vivo experiments that CitB performs a benzylic hydroxylation in the biosynthesis of the mycotoxin citrinin (8, Fig. 1B). ${ }^{38} \mathrm{ClaD}$ was shown by Li and coworkers to perform a similar transformation in the biosynthesis of peniphenones and penilactones (Fig. 1B, 9-11). ${ }^{17}$ We envisioned employing CitB and $\mathrm{ClaD}$ as general catalysts for benzylic hydroxylation of ortho-cresol substrates. We anticipated that, following benzylic $\mathrm{C}-\mathrm{H}$ bond oxidation, an $o-\mathrm{QM}$ intermediate (see 12-14 Fig. 1C) could be accessed and intercepted with a variety of nucleophiles or dienophiles to form benzylic $\mathrm{C}-\mathrm{O}, \mathrm{C}-\mathrm{N}, \mathrm{C}-\mathrm{S}$, and $\mathrm{C}-\mathrm{C}$ bonds in a biomimetic fashion. ${ }^{17}$ Here, we demonstrate this approach with two biocatalysts, but we anticipate this chemoenzymatic platform could be executed with a plethora of wild-type enzymes to access more diverse substrates and complementary selectivity.

\section{RESULTS AND DISCUSSION}

Toward developing a chemoenzymatic method for benzylic $\mathrm{C}-\mathrm{H}$ functionalization, we tested the feasibility of biocatalytic $\mathrm{C}-\mathrm{H}$ hydroxylation, coupled with $o-\mathrm{QM}$ formation and derivatization with a model substrate (see 16, Fig. 2) that captures the conserved methyl resorcinol core of CitB and ClaD's native substrates. This model compound maintained the redox-sensitive aldehyde moiety and hydroxyl groups present in the citrinin biosynthetic intermediate (7). This model compound, 16, was completely consumed in $3 \mathrm{~h}$ upon exposure to $0.4 \mathrm{~mol} \% \mathrm{CitB}$ in the presence of $\alpha-\mathrm{KG}$, an iron(II) source and sodium ascorbate in $50 \mathrm{mM}$ TES buffer at pH 7.5 (Fig. 2). From this reaction, a single benzylic alcohol product (17) was isolated in $82 \%$ yield without any evidence of oxidation of either the aldehyde or the resorcinol core. This result demonstrated that CitB could function on a non-native substrate with the robustness needed for preparative-scale synthesis with precise chemo- and site-selectivity. As a comparative measure, we subjected substrate $\mathbf{1 6}$ to several chemical oxidation conditions and did not observe conversion to desired benzylic alcohol 17 (see Supporting Information, Table S1). For example, attempts to oxidize 16 with $\mathrm{DDQ}^{39}$ resulted in over-oxidation to the bisaldehdye and exposure of $\mathbf{1 6}$ to $\mathrm{MnO}_{2}{ }^{40}$ or $\mathrm{Ag}_{2} \mathrm{O}^{41}$ resulted in no observed reaction. Efforts to perform a benzylic hydroxylation with $\mathrm{K}_{2} \mathrm{~S}_{2} \mathrm{O}_{8}{ }^{42}$ or cerium ammonium nitrate ${ }^{43}$ led to decomposition of the substrate. Radical bromination with AIBN/NBS resulted in aromatic bromination and did not generate the desired benzylic halide for subsequent hydrolysis to the benzyl alcohol. ${ }^{44}$ These results capture the challenge in accomplishing this seemingly simple benzylic hydroxylation in the presence of redox-sensitive aldehyde and phenolic groups, as well as the advantages provided by biocatalytic benzylic $\mathrm{C}-\mathrm{H}$ hydroxylation. Next, the reactivity

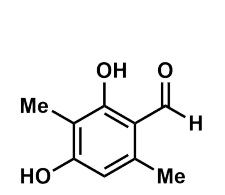

16
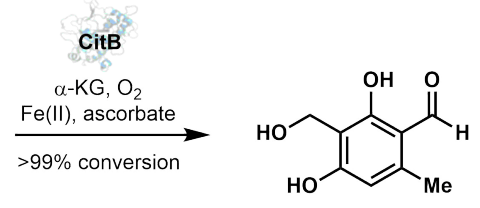

17

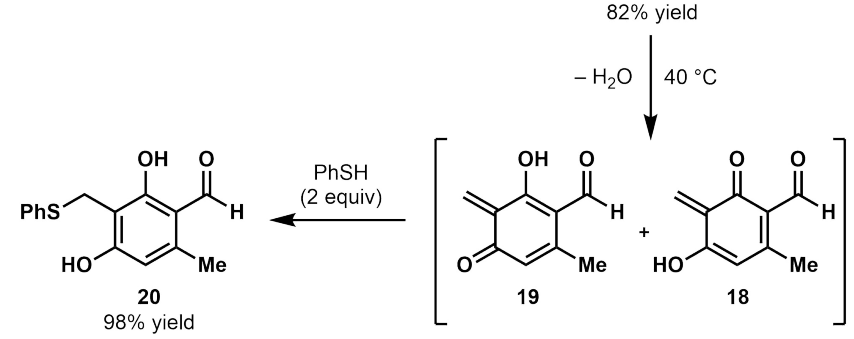

Figure 2. Initial experiments to assess feasibility of NHI biocatalyst-initiated ortho-quinone methide formation and functionalization.

of benzylic alcohol 17 was investigated under aqueous conditions. Gratifyingly, upon gentle heating in the presence of thiophenol, alcohol $\mathbf{1 7}$ was quantitatively transformed into thioether 20 through an anticipated $o-\mathrm{QM}$ intermediate (18-19). These experiments provided evidence for $o$-QM formation under remarkably mild conditions compared to previous reports for the generation of $o$-QMs from ortho-hydroxy benzylic alcohols. ${ }^{4}, 45-46$

Evidence that CitB is sufficiently robust to perform preparative-scale reactions and provide access to $o$-QMs under mild conditions spurred further investigation of this chemoenzymatic strategy for benzylic functionalization, including studies with the homologous NHI enzyme, ClaD. To investigate the substrate scope of CitB and ClaD, a panel of substrates was synthesized possessing an ortho-cresol core (Fig. 3). ${ }^{47}$ This panel was designed to define the electronic and steric parameters of substrates that undergo a productive reaction with the NHI biocatalysts. Compounds 9, 16, and 23-42 were subjected to analytical-scale reactions with CitB and ClaD, using standard conditions for $\alpha$-KGdependent non-heme iron oxygenases. ${ }^{37}$ These analyticalscale reactions were performed in vitro with purified enzyme (Supporting Information, Fig. S5), as well as in whole cell or crude cell lysate format (Fig. 3). For CitB, a whole cell reaction platform was shown to affect benzylic $\mathrm{C}-\mathrm{H}$ hydroxylation in an efficient manner. However, whole cell reactions were not effective for $\mathrm{ClaD}$, leading us to perform reactions using filtered crude cell lysate. Both of these reaction platforms provide an operationally-simple method for preparing and using NHI biocatalysts in a manner that is amenable to preparative-scale reactions, avoiding arduous protein purification steps. ${ }^{48-50}$ Analytical-scale reactions revealed that $\mathrm{CitB}$ and $\mathrm{ClaD}$ each selectively hydroxylate compounds with a variety of steric and electronic properties (Fig. 3). Introducing bulky, electron-withdrawing or electron-donating groups at either the $\mathrm{C} 5$ or $\mathrm{C} 6$ position did not prevent productive reactions with CitB. However, ClaD demonstrated limitations in its ability to accept larger groups, such as phenyl substituents at C5 and C6. The complementarity of substrate scopes between CitB and ClaD is also observed in the substitution at C4. For CitB, the formyl group at $\mathrm{C} 4$ can be substituted with alternative electronwithdrawing substituents such as a nitro group (25) or 
imine (24). However, substrates with greater steric bulk at $\mathrm{C} 4$, such as ketone substrates, were generally not hydroxylated by $\mathrm{CitB}$. In contrast, $\mathrm{ClaD}$ can productively react with a variety of ketones (see 9, 27-30). The importance of this C4 substituent is also highlighted by the lack of reaction observed with 2,5-dimethylresorcinol (23). Based on preliminary analysis of models of these biocatalysts, we anticipate that a hydrogen bond acceptor is critical at the $C 4$ position to achieve a catalytically active conformation of the substrate-enzyme complex. Current efforts are focused on obtaining engineered enzyme variants that do not require substrates bearing a hydrogen bond acceptor, such as an aldehyde, for a productive reaction to occur. To probe the substrate scope of our biocatalysts beyond resorcinol substrates, phenolic compounds $\mathbf{3 9}$ and $\mathbf{S 5}$ (see Supporting Information) were subjected to CitB hydroxylation conditions. Phenol 39 was converted to the corresponding benzylic alcohol; however, the position of the hydroxyl group proved to be important for catalysis as phenol S5 was not oxidized by either CitB or ClaD. These results motivated us to synthesize an additional panel of phenolic substrates to further assess the scope of NHI biocatalytic hydroxylation (40-42). In the case of CitB, increasing the steric bulk at the C5 position increased the conversion of substrates to hydroxylated products except for 2-naphthyl substrate 42, which demonstrated decreased conversion, possibly reflecting the steric limitations of the CitB active site. ClaD was unable to hydroxylate phenolic substrates 40-42.

As a next step in the evaluation of the synthetic utility of the NHI dioxygenase-catalyzed benzylic C-H hydroxylation, we performed reactions on preparative-scale using whole cell (CitB) and crude cell lysate (ClaD) conditions (Fig. 4). This method enabled routine performance of reactions on $>500 \mathrm{mg}$ scale without the requirement for protein purification. Isolation and characterization of C5-substituted benzylic alcohol products 17 and 46-48 was achieved with yields that corresponded to starting material consumption. For example, benzylic alcohol 17 was isolated in $82 \%$ yield. However, poor isolated yields were obtained for substrates with C6-substituents, despite nearly complete consumption of starting material (see 49-53). Further analysis of the crude product mixture from these reactions revealed a second product, in which a molecule of ascorbic acid had been incorporated, presumably through interception of an intermediate $o$-QM (see Supporting Information Fig. S63).

The observed reactivity of C6-substituted alcohols was explored computationally and can be explained by thermodynamics of the reaction sequence of $\mathbf{4 3}$ to $\mathbf{4 5}$ (Fig. 4A), which includes hypothetical substrates to explore the electronic effects at both the C5 and C6 positions (see Supporting Information Part XIV for detailed analysis). We identified a relationship between inductive effects and Gibbs free energy. The thermodynamic trends indicated that benzylic alcohols with electron-withdrawing substituents at the C6position were less thermodynamically stable, making these substrates more reactive and prone to generation of an $o$ QM. This computational data supported our experimental observations. Therefore, we hypothesized that higher yields could be achieved if the reactive benzylic alcohol products were first transformed into more stable benzylic thioethers by interception of intermediate $o-\mathrm{QMs}$ with thiophenol. The corresponding thermodynamic analysis of thiophenol adducts 49-53 revealed that, regardless of substitution, the benzylic thioethers are lower in energy than their respective benzylic alcohol precursor (Fig. 4C and Supporting Information Figs. S88 and S89). To test this hypothesis, we developed an in situ functionalization protocol, demonstrating that increasing the reaction temperature to $40{ }^{\circ} \mathrm{C}$ and adding two equivalents of thiophenol led to the isolation of the desired benzylic thioethers 49-53 and 62 as the sole product in each reaction.

Motivated by this initial success in generating and diversifying $o$-QMs under mild conditions, we assessed the range of products accessible through this biocatalyst-initiated cascade. First, a panel of nucleophiles was evaluated in 1,4additions to the in situ-generated o-QMs (Fig. 5, 18 and 19). Both linear and branched alcohols were competent nucleophiles, affording the desired products (73-75) in good yields. A secondary amine nucleophile was also well tolerated (77); however, reactions with several primary amines resulted in an undesired condensation reaction with the pendant aldehyde to form imines. Thiol nucleophiles reacted smoothly to provide the desired conjugate addition products (20 and 76) in excellent yields. The comparatively high yield of thioether adducts relative to alkyl ether adducts is likely due to the increased nucleophilicity of sulfur as well as stability of the sulfur adducts compared to the alcohol precursor.

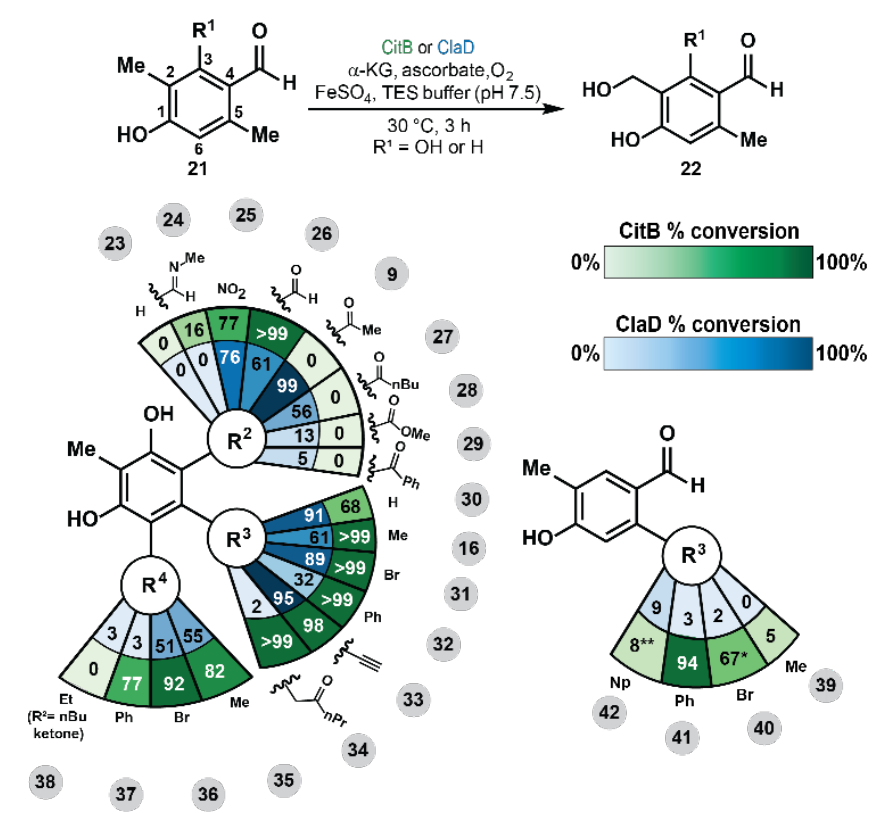

Figure 3. Substrate scope for CitB and ClaD-catalyzed benzylic $\mathrm{C}-\mathrm{H}$ hydroxylation. Reaction conditions: $2.5 \mathrm{mM}$ substrate, $45 \mathrm{mg} / \mathrm{mL}$ CitB wet cell pellet or $10 \% \mathrm{v} / \mathrm{v}$ ClaD crude cell lysate, $50 \mathrm{mM}$ TES pH 7.5, $5 \mathrm{mM} \alpha$-ketoglutaric acid $(\alpha$ $\mathrm{KG}$ ), $8 \mathrm{mM}$ sodium ascorbate (NaAsc), $0.1 \mathrm{mM}$ ferrous sulfate (FeSO 4 ), $30^{\circ} \mathrm{C}, 100 \mathrm{rpm}$ shaking, $3 \mathrm{~h}$. [ $\left.{ }^{*}\right]$ with $15 \%$ acetonitrile as cosolvent. [**] with $15 \%$ tetrahydrofuran as cosolvent. Conversion to product was quantified by UPLC-DAD analysis. 

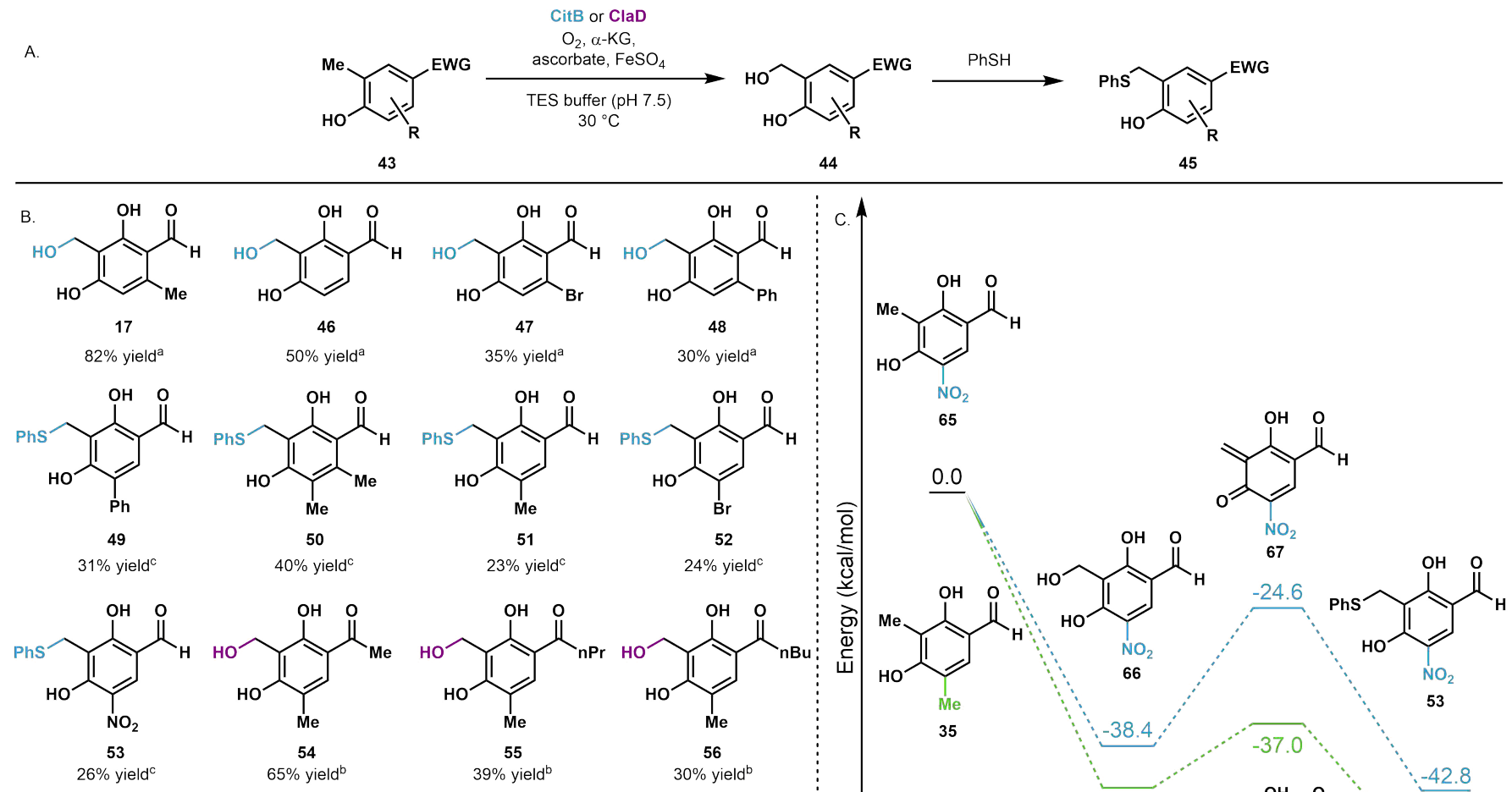

44

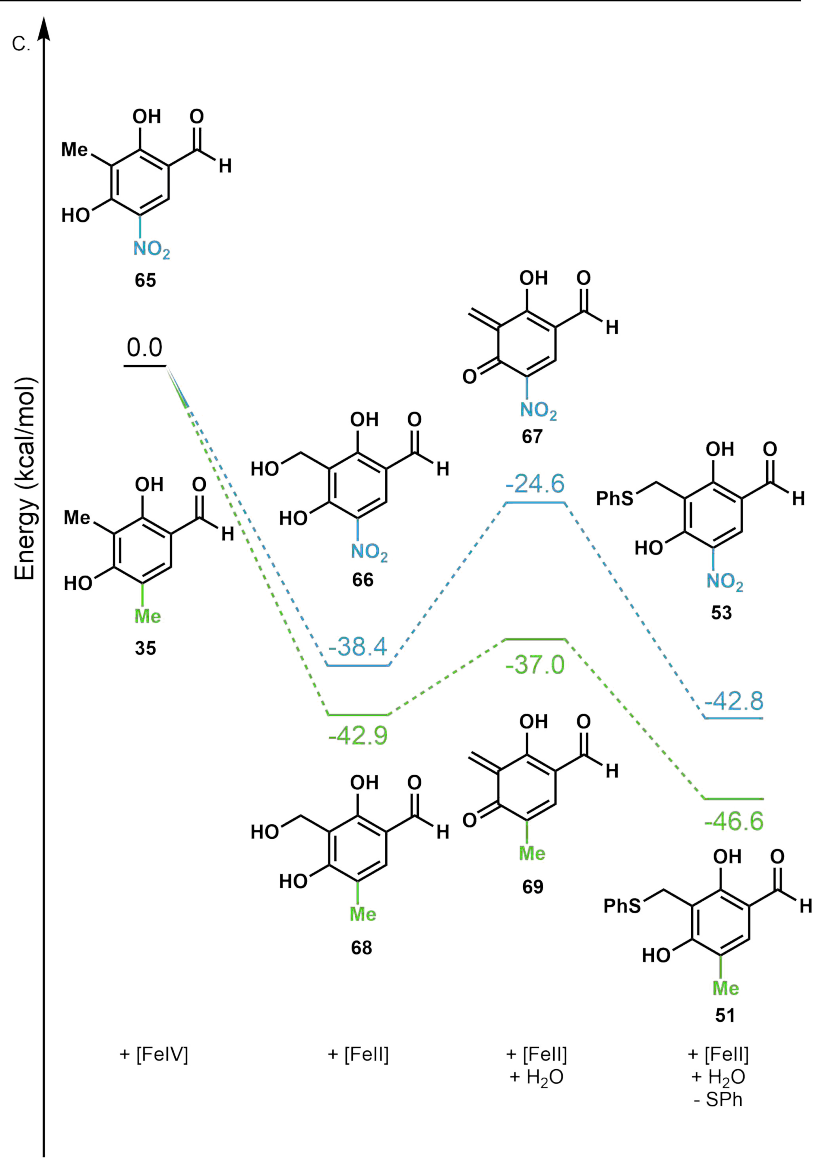

Figure 4. A. General scheme for CitB and ClaD-catalyzed benzylic hydroxylation and in situ functionalization with thiophenol. B. Preparative scale reaction isolated yields for CitB-catalyzed hydroxylation and functionalization. Reaction conditions: $2.5 \mathrm{mM}$ substrate, [a] $45 \mathrm{mg} / \mathrm{mL}$ CitB wet cell pellet or [b]10\% v/v ClaD clarified cell lysate, $50 \mathrm{mM}$ TES pH 7.5, $5 \mathrm{mM} \alpha$-ketoglutaric acid $(\alpha-\mathrm{KG}), 8 \mathrm{mM}$ sodium ascorbate ( $\mathrm{NaAsc}), 0.1 \mathrm{mM}$ ferrous sulfate $\left(\mathrm{FeSO}_{4}\right), 30^{\circ} \mathrm{C}, 100 \mathrm{rpm}$ shaking, $3 \mathrm{~h}$. [c] PhSH was added directly to reaction mixture after conversion to benzylic alcohol and incubated at $40^{\circ} \mathrm{C}, 3 \mathrm{~h}, 100 \mathrm{rpm}$ shaking. C. Thermodynamic analysis of C6-methyl (green) and C6-nitro (blue) substrates. Structures represent the starting material, benzylic alcohol product, $o$-QM, and thiophenol adducts (left to right). The energies are mass balanced with a truncated 2-His-1-Asp non-heme iron system (see Supporting Information Fig. S86). Geometry optimizations and frequency calculations were performed at B3LYP 6-311++G** and $6-31 \mathrm{G}^{* *}$ for iron.

The feasibility of benzylic $\mathrm{C}-\mathrm{C}$ bond formation was probed through inverse electron-demand Diels-Alder (IEDDA) reactions. The reactivity of biocatalyst-generated benzyl alcohol 17 with various dienophiles was evaluated through a one-pot reaction sequence. Following generation of 17 by CitB, the reaction mixture was heated to $45^{\circ} \mathrm{C}$ in the presence of ethyl vinyl ether. Gratifyingly, chroman products 78 and 79 were produced in $64 \%$ and $27 \%$ yield, respectively. Reactions with electron-rich dienophiles (7883) proceeded smoothly to deliver mixtures of two isomeric products derived from the two possible $o-\mathrm{QM}$ intermediates (18 and 19). Reactions employing electron-rich dienophiles typically afforded 4:1 mixtures of isomeric products, favoring the product derived from reaction with the C1 $o-Q M(19)$. Reactions with styrenes 84-89 were also carried out. Due to the electron-deficient character of these dienophiles, these reactions required a higher temperature, $65{ }^{\circ} \mathrm{C}$, to undergo a productive reaction. The major products of these IEDDA reactions resulted from reaction with the $o$ QM formed at the C3 hydroxyl group (18), while the minor isomers were formed from reaction with the C $10-\mathrm{QM}$ species (19). This operationally-simple, one-pot protocol accomplishes the direct conversion of benzylic $\mathrm{C}-\mathrm{H}$ bonds to $\mathrm{C}-\mathrm{O}, \mathrm{C}-\mathrm{N}, \mathrm{C}-\mathrm{S}$ and $\mathrm{C}-\mathrm{C}$ bonds, allowing for direct access to diverse chemical scaffolds and demonstrating the synthetic utility of this biocatalyst.

Upon the observation that $o$-QM generation occurs at reduced temperatures, we hypothesized that these reactions likely proceed through low barrier processes. This was assessed and confirmed computationally using benzylic alcohol 17. We generated models for both $\mathrm{C} 1$ and $\mathrm{C} 3 \mathrm{o}-\mathrm{QM}$ s and 
A.
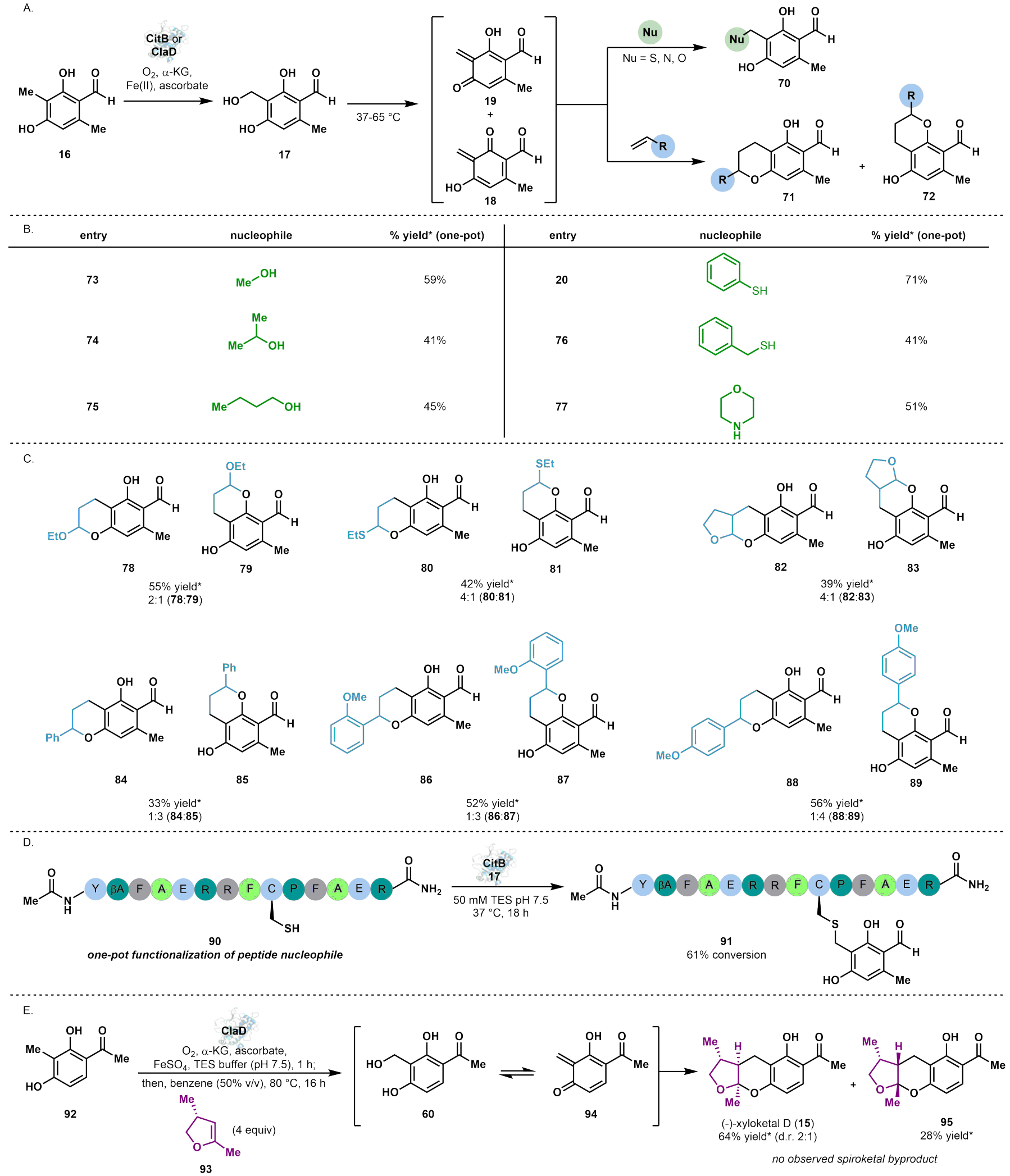

Figure 5. One-pot NHI biocatalyst-initiated $o$-QM generation and diversification. A. Yields of one-pot Michael addition reactions. B. Yields of one-pot IEDDA reactions. C. One-pot functionalization of cysteine-containing peptide. D. One-pot chemoenzymatic synthesis of (-)-xyloketal D (15). HPLC yields determined by analysis of isolated product standard curves.

modelled the subsequent IEDDA reactions (Supporting Information Fig. S82). In this model, $o$-QM generation was found to proceed through a concerted, low barrier transition state, while reactions with ethyl vinyl ether and styrene were found to proceed through concerted, asynchronous transition states that are typically proposed for IEDDA mechanisms (Supporting Information Figs. S83 and S84). 
Additionally, we envisioned that this method could be extended to the functionalization of biomolecules under physiological conditions to selectively modify cysteine residues in peptides and proteins. Two complementary peptides were synthesized: one peptide that contained a cysteine residue (90) and another in which the cysteine was replaced with a lysine residue (S14). Substrate 16 was subjected to in vitro CitB-catalyzed hydroxylation conditions in the presence of either peptide 90 or S14. After an $18 \mathrm{~h}$ incubation the cysteine-containing peptide was converted to singly-modified peptide 91 (61\% conversion). Under the same conditions, the peptide containing a lysine in place of cysteine (S14) was not modified through imine condensation or through addition to the $o-\mathrm{QM}$. These cascade reactions demonstrate the utility of this biocatalytic method in providing a platform for in situ functionalization of peptides.

Finally, to demonstrate the utility of this biocatalytic method for the synthesis of a secondary metabolite, we aimed to develop a chemoenzymatic route to the chroman natural product (-)-xyloketal D (15). Xyloketal D is a fungal natural product from Xylaria sp. found in the South China Sea. ${ }^{51}$ Synthetic strategies have been developed previously for the synthesis of (-)-xyloketal D including a Diels-Alder cycloaddition by Wilson and co-workers, ${ }^{7}$ a sequential $\mathrm{Mi}$ chael-addition ketalization sequence by Flörke and coworkers ${ }^{52}$ and, more recently, a gold-catalyzed cycloisomerization reported by Sarkar and co-workers in 2013.53 The latter two syntheses report high diastereoselectivity (19:2 and $>20: 1$, respectively); however, the Michael-addition ketalization yields a mixture of regioisomers and the goldcatalyzed cycloisomerization requires a lengthy precursor synthesis. The approach developed by Wilson and co-workers provided the required $o-\mathrm{QM}$ precursor in a single step; however, their conditions for $o$-QM generation led to a low overall yield due in part to undesired isomerization of the dienophile under the requisite conditions for $o-\mathrm{QM}$ formation. Ultimately, this approach provided a 2:1 mixture of the desired chroman ketal and undesired chroman spiroketal products. ${ }^{7}$ Our chemoenzymatic strategy controls the site-selectivity of the oxidation and provides a more active $o$-QM precursor (see Supporting Information Fig. S93) allowing for more mild cycloaddition conditions and circumventing any dienophile isomerization challenges. After synthesis of chiral dienophile 93, according to Wilson's established procedure, we subjected methyl ketone 92 to our conditions for biocatalytic benzylic $\mathrm{C}-\mathrm{H}$ hydroxylation with ClaD crude lysate, obtaining complete conversion to benzylic alcohol 60. Following the enzymatic $\mathrm{C}-\mathrm{H}$ benzylic hydroxylation step, benzene was added directly to the biocatalytic reaction mixture, along with dienophile $\mathbf{9 3}$. The reaction tube was sealed and heated to $80^{\circ} \mathrm{C}$ for $16 \mathrm{~h}$. This process generated (-)-xyloketal D (15) in a 64\% yield (2:1 ratio) with the diastereomer $(\mathbf{9 5}, 28 \%$ yield) and no detectable formation of unwanted spiroketal products. Thus, our approach enabled a one-pot chemoenzymatic synthesis of (-)-xyloketal D (15), with improved yields and under milder conditions compared to a previous $o$-QM-based approach to this molecule. ${ }^{7} \mathrm{We}$ anticipate this one-pot chemoenzymatic strategy will facilitate the streamlined synthesis of a variety of natural products.

\section{CONCLUSIONS}

We have demonstrated the utility of biocatalysis for chemoand site-selective $\mathrm{C}-\mathrm{H}$ functionalization through NHI biocatalyst-initiated benzylic functionalization of ortho-cresol substrates. This was accomplished by establishing scalable biocatalytic reaction methods to provide an inexpensive and highly accessible platform for benzylic hydroxylation. Using this platform, we have leveraged NHI dioxygenasecatalyzed hydroxylation for further diversification of phenolic scaffolds. Chemoenzymatic formation of $o-\mathrm{QM}$ intermediates was followed by reactions with small-molecule and peptide nucleophiles as well as electron-rich dienophiles, leading to the synthesis of the fungal natural product $(-)$-xyloketal D. Thus, direct access to a diverse set of compounds though this simple chemoenzymatic cascade is possible. We anticipate that our method for biocatalyst-initiated $o$-QM generation can be broadly applied to other chemoenzymatic systems capable of performing benzylic $\mathrm{C}-\mathrm{H}$ hydroxylation and will enable transformations complementary to small molecule catalysts and reagents.

\section{ASSOCIATED CONTENT}

Supporting Information. Experimental details, NMR spectra, full compound characterization, UPLC and LCMS traces, details of protein expression and purification and peptide synthesis. This material is available free of charge via the Internet at http://pubs.acs.org.

\section{AUTHOR INFORMATION}

Corresponding Author

*arhardin@umich.edu

\section{Author Contributions}

[+] These authors contributed equally.

\section{Notes}

The authors declare no competing financial interest.

\section{ACKNOWLEDGMENT}

This work was supported by funds from the University of Michigan Life Sciences Institute and Department of Chemistry. T.J.D. thanks the National Science Foundation for a Graduate Research Fellowship. S.A.B.D. acknowledges a National Institutes of Health Chemistry Biology Interface Training Grant (T32 GM 008597). K.C.S acknowledges a Rackham Merit Fellowship from the University of Michigan Rackham Graduate School. P.M.Z. acknowledges partial support from NIH R35GM128830. The authors thank Dr. Steven Sturlis (University of Michigan) for his assistance in peptide synthesis and purification. The authors also thank Shuri Francis (University of Michigan) for her assistance in substrate synthesis and Attabey Rodríguez Benítez for her assistance in figure design. 


\section{ABBREVIATIONS}

TES: N-[Tris(hydroxymethyl)methyl]-2-aminoethanesulfonic acid, UPLC: ultra performance liquid chromatography, LCMS: liquid chromatography mass spectrometry.

\section{REFERENCES}

1. Ueberbacher, B. T.; Hall, M.; Faber, K., Electrophilic and nucleophilic enzymatic cascade reactions in biosynthesis. Nat. Prod. Rep. 2012, 29 (3), 337-50.

2. Hashimoto, T.; Kuzuyama, T., Mechanistic insights into Diels-Alder reactions in natural product biosynthesis. Curr. Opin. Chem. Biol. 2016, 35, 117-123.

3. Bai, W. J.; David, J. G.; Feng, Z. G.; Weaver, M. G.; Wu, K. L.; Pettus, T. R., The domestication of ortho-quinone methides. Acc. Chem. Res. 2014, 47 (12), 3655-64.

4. Singh, M. S.; Nagaraju, A.; Anand, N.; Chowdhury, S., ortho-Quinone methide (o-QM): a highly reactive, ephemeral and versatile intermediate in organic synthesis. RSC Adv. 2014, 4 (99), 55924 55959.

5. Van De Water, R. W.; Pettus, T. R. R., o-Quinone methides: intermediates underdeveloped and underutilized in organic synthesis. Tetrahedron 2002, 58 (27), 5367-5405.

6. Willis, N. J.; Bray, C. D., ortho-Quinone Methides in Natural Product Synthesis. Chemistry - A European Journal 2012, 18 (30), 9160-9173.

7. Pettigrew, J. D.; Freeman, R. P.; Wilson, P. D., Total synthesis of ()-xyloketal D and its enantiomer: Confirmation of absolute stereochemistry. Can. J. Chem. 2004, 82 (11), 1640-1648.

8. Spence, J. T.; George, J. H., Total Synthesis of Peniphenones A-D via Biomimetic Reactions of a Common o-Quinone Methide Intermediate. Org. Lett. 2015, 17 (24), 5970-3.

9. Spence, J. T. J.; George, J. H., Biomimetic Total Synthesis of entPenilactone A and Penilactone B. Org. Lett. 2013, 15 (15), 38913893.

10. Basha, R. S.; Chen, C. W.; Reddy, D. M.; Lee, C. F., Iodine-Mediated Direct Generation of o-Quinone Methides at Room Temperature: A Facile Protocol for the Synthesis of ortho-Hydroxybenzyl Thioethers. Chem. Asian J. 2018, 13 (17), 2475-2483.

11. Zhou, D.; Yu, X.; Zhang, J.; Wang, W.; Xie, H., Organocatalytic Asymmetric Formal [4 + 2] Cycloaddition of in Situ OxidationGenerated ortho-Quinone Methides and Aldehydes. Org. Lett. 2018, 20 (1), 174-177.

12. Reichl, K. D.; Smith, M. J.; Song, M. K.; Johnson, R. P.; Porco, J. A., Jr., Biomimetic Total Synthesis of (+/-)-Griffipavixanthone via a Cationic Cycloaddition-Cyclization Cascade. J. Am. Chem. Soc. 2017, 139 (40), 14053-14056.

13. More, A. A.; Ramana, C. V., o-Quinone Methides via OxoneMediated Benzofuran Oxidative Dearomatization and Their Intramolecular Cycloaddition with Carbonyl Groups: An Expeditious Construction of the Central Tetracyclic Core of Integrastatins, Epicoccolide A, and Epicocconigrone A. Org. Lett. 2016, 18 (3), 612-615

14. Gebauer, K.; Reuss, F.; Spanka, M.; Schneider, C., Relay Catalysis: Manganese(III) Phosphate Catalyzed Asymmetric Addition of betaDicarbonyls to ortho-Quinone Methides Generated by Catalytic Aerobic Oxidation. Org Lett 2017, 19 (17), 4588-4591.

15. Wong, Y. F.; Wang, Z.; Hong, W.-X.; Sun, J., A one-pot oxidation/cycloaddition cascade synthesis of 2,4-diaryl chromans via ortho -quinone methides. Tetrahedron 2016, 72 (22), 27482751

16. Lam, H. C.; Spence, J. T.; George, J. H., Biomimetic Total Synthesis of Hyperjapones A-E and Hyperjaponols A and C. Angew. Chem. Int. Ed. Engl. 2016, 55 (35), 10368-71.

17. Fan, J.; Liao, G.; Kindinger, F.; Ludwig-Radtke, L.; Yin, W. B.; Li, S. M., Peniphenone and Penilactone Formation in Penicillium crustosum via 1,4-Michael Additions of ortho-Quinone Methide from Hydroxyclavatol to gamma-Butyrolactones from Crustosic Acid. J. Am. Chem. Soc. 2019, 141, (10), 4225-4229.

18. Lie, F.; Chen, Y.; Wang, Z.; Li, Z., Enantioselective benzylic hydroxylation of indan and tetralin with Pseudomonas monteilii TA5. Tetrahedron: Asymmetry 2009, 20 (10), 1206-1211.

19. Yongzheng, C.; Felicia, L.; Zhi, L., Enantioselective Benzylic Hydroxylation with Pseudomonas monteilii TA-5: A Simple Method for the Syntheses of (R)-Benzylic Alcohols Containing Reactive Functional Groups. Adv. Synth. \& Catal. 2009, 351 (13), 2107-2112. 20. Limberger, R. P.; Ursini, C. V.; Moran, P. J. S.; Rodrigues, J. A. R., Enantioselective benzylic microbial hydroxylation of indan and tetralin. J. Mol. Catal. B: Enzym. 2007, 46 (1), 37-42.

21. Dai, S.; Wu, J.; Wang, Z.; Chen, Y.; Li, Z., Highly chemo- and regioselective hydroxylations of o- and m-substituted toluenes to benzyl alcohols with Cellulosimicrobium cellulans EB-8-4. Tetrahedron 2010, 66 (34), 6919-6923.

22. Uzura, A.; Katsuragi, T.; Tani, Y., Stereoselective oxidation of alkylbenzenes by fungi. J. Biosci. Bioengin. 2001, 91 (2), 217-221.

23. Yadav, S.; Yadav, R. S. S.; Yadava, S.; Yadav, K. D. S., Stereoselective hydroxylation of ethylbenzene to (R)-1-phenylethanol using mycelia of Aspergillus niger as catalyst. Catal. Commun. 2011, 12 (9), 781-784.

24. Alexander, D.; Maria, W. A.; Tsvetan, K.; Andrea, M. C.; Erika, T.; Martin, S.; Joëlle, R. A.; Ulrich, S., An Enzymatic Route to $\alpha$ Tocopherol Synthons: Aromatic Hydroxylation of Pseudocumene and Mesitylene with P450 BM3. Chem. Eur. J. 2017, 23 (71), 1798117991.

25. Anja, E.; Łukasz, G.; Susanne, H.; P., K. P.; J., T. N.; Jürgen, P.; L., F. S., Enantioselective Benzylic Hydroxylation Catalysed by P450 Monooxygenases: Characterisation of a P450cam Mutant Library and Molecular Modelling. ChemBioChem 2016, 17 (5), 426-432.

26. Driscoll, J. P.; Kornecki, K.; Wolkowski, J. P.; Chupak, L.; Kalgutkar, A. S.; O'Donnell, J. P., Bioactivation of Phencyclidine in Rat and Human Liver Microsomes and Recombinant P450 2B Enzymes: Evidence for the Formation of a Novel Quinone Methide Intermediate. Chem. Res. Toxicol. 2007, 20 (10), 1488-1497.

27. Du, L.; Dong, S.; Zhang, X.; Jiang, C.; Chen, J.; Yao, L.; Wang, X.; Wan, X.; Liu, X.; Wang, X.; Huang, S.; Cui, Q.; Feng, Y.; Liu, S.-J.; Li, S., Selective oxidation of aliphatic $\mathrm{C}-\mathrm{H}$ bonds in alkylphenols by a chemomimetic biocatalytic system. Proc. Nat'l. Acad. Sci. 2017, 114 (26), E5129-E5137.

28. Kelly, P. P.; Eichler, A.; Herter, S.; Kranz, D. C.; Turner, N. J.; Flitsch, S. L., Active site diversification of P450cam with indole generates catalysts for benzylic oxidation reactions. Beilstein J. Org. Chem. 2015, 11, 1713-1720.

29. Li, A.; Wu, S.; Adams, J. P.; Snajdrova, R.; Li, Z., Asymmetric epoxidation of alkenes and benzylic hydroxylation with P450tol monooxygenase from Rhodococcus coprophilus TC-2. Chem. Commun. 2014, 50 (63), 8771-8774.

30. Neufeld, K.; Marienhagen, J.; Schwaneberg, U.; Pietruszka, J., Benzylic hydroxylation of aromatic compounds by P450 BM3. Green Chem. 2013, 15 (9), 2408-2421.

31. Suzuki, K.; Stanfield, J. K.; Shoji, O.; Yanagisawa, S.; Sugimoto, H.; Shiro, Y.; Watanabe, Y., Control of stereoselectivity of benzylic hydroxylation catalysed by wild-type cytochrome P450BM3 using decoy molecules. Catal. Sci. \& Technol. 2017, 7 (15), 3332-3338.

32. Hall, E. A.; Sarkar, M. R.; Bell, S. G., The selective oxidation of substituted aromatic hydrocarbons and the observation of uncoupling via redox cycling during naphthalene oxidation by the CYP101B1 system. Catal. Sci. \& Technol. 2017, 7 (7), 1537-1548. 33. Zwick, C. R.; Renata, H., Evolution of Biocatalytic and Chemocatalytic C-H Functionalization Strategy in the Synthesis of Manzacidin C. J. Org. Chem. 2018, 83 (14), 7407-7415.

34. Dror, A.; Fishman, A., Engineering non-heme mono- and dioxygenases for biocatalysis. Comput. Struct. Biotechnol. J. 2012, 2 (3), e201209011.

35. Herr, C. Q.; Hausinger, R. P., Amazing Diversity in Biochemical Roles of Fe(II)/2-Oxoglutarate Oxygenases. Trends Biochem. Sci. 2018. 
36. Martinez, S.; Hausinger, R. P., Catalytic Mechanisms of Fe(II)and 2-Oxoglutarate-dependent Oxygenases. J. Biol. Chem. 2015, 290 (34), 20702-11.

37. Nakamura, H.; Matsuda, Y.; Abe, I., Unique chemistry of nonheme iron enzymes in fungal biosynthetic pathways. Nat. Prod. Rep. 2018, 35 (7), 633-645.

38. He, Y.; Cox, R. J., The molecular steps of citrinin biosynthesis in fungi. Chem. Sci. 2016, 7 (3), 2119-2127.

39. Takuya, M.; Pal, S. I.; Hideo, E.; Hitoshi, T., The First Total Synthesis of Grandinal, a New Phloroglucinol Derivative Isolated from Eucalyptus grandis. Chem. Lett. 2001, 30 (3), 210-211.

40. Carter, D. V.; Charlton, P. T.; Fenton, A. H.; Housley, J. R.; Lessel, B., The preparation and the antibacterial and antifungal properties of some substituted benzyl alcohols. J. Pharm. Pharmacol. 1958, 10 (S1), 149T-159T.

41. Li, L.; Liu, Y.; Wang, Q., Regioselective Oxidative Dehydrogenation under Nonenzymatic Conditions: A Synthetic Route to Gossypol. Eur. J. Org. Chem. 2013, 2013 (35), 8014-8021. 42. Cort, A. D.; Mandolini, L.; Panaioli, S., Selective One-Pot Oxidation of Methylarenes to Benzyl Alcohols with the Copper(II)Peroxydisulfate System. Synth. Commun. 1988, 18 (6), 613-616.

43. Baciocchi, E.; Mandolini, L.; Rol, C., Oxidation by metal ions. 6. Intramolecular selectivity in the side-chain oxidation of p-ethyltoluene and isodurene by cobalt(III), cerium(IV), and manganese(III). J. Org. Chem. 1980, 45 (19), 3906-3909.

44. Vece, V.; Jakkepally, S.; Hanessian, S., Total Synthesis and Absolute Stereochemical Assignment of the Insecticidal Metabolites Yaequinolones J1 and J2. Org. Lett. 2018, 20 (14), 4277-4280.

45. Yang, B.; Gao, S., Recent advances in the application of DielsAlder reactions involving $o$-quinodimethanes, aza-o-quinone methides and $o$-quinone methides in natural product total synthesis. Chem. Soc. Rev. 2018, 47 (21), 7926-7953.

46. Arumugam, S.; Popik, V. V., Photochemical Generation and the Reactivity of o-Naphthoquinone Methides in Aqueous Solutions. J. Am. Chem. Soc. 2009, 131 (33), 11892-11899.

47. Baker Dockrey, S. A.; Lukowski, A. L.; Becker, M. R.; Narayan, A. R. H., Biocatalytic site- and enantioselective oxidative dearomatization of phenols. Nat. Chem. 2017, 10, 119.

48. de Carvalho, C. C. C. R., Whole cell biocatalysts: essential workers from Nature to the industry. Microb. Biotechnol. 2017, 10 (2), 250-263.

49. Wachtmeister, J.; Rother, D., Recent advances in whole cell biocatalysis techniques bridging from investigative to industrial scale. Curr. Opin. Biotechnol. 2016, 42, 169-177.

50. Baker Dockrey, S. A.; Doyon, T. J.; Perkins, J. C.; Narayan, A. R. H., Whole-cell biocatalysis platform for gram-scale oxidative dearomatization of phenols. Chem. Biol. \& Drug Des. 2018, 93, 1207-1213.

51. Lin, Y.; Wu, X.; Feng, S.; Jiang, G.; Luo, J.; Zhou, S.; Vrijmoed, L. L. P.; Jones, E. B. G.; Krohn, K.; Steingröver, K.; Zsila, F., Five Unique Compounds: Xyloketals from Mangrove Fungus Xylaria sp. from the South China Sea Coast. The Journal of Organic Chemistry 2001, 66 (19), 6252-6256.

52. Krohn, K.; Riaz, M.; Flörke, U., Synthesis of Xyloketals, Natural Products from the Mangrove Fungus Xylaria sp. European Journal of Organic Chemistry 2004, 2004 (6), 1261-1270.

53. Panda, B.; Sarkar, T. K., Gold catalysis: regio- and stereoselective total synthesis of xyloketals D and $\mathrm{G}$ and the related natural product alboatrin. J Org Chem 2013, 78 (6), 2413-21. 


$$
=
$$

